\title{
Bravo 48-hour Wireless pH Monitoring in Patients With Non-cardiac Chest Pain. Objective Gastroesophageal Reflux Disease Parameters Predict the Responses to Proton Pump Inhibitors
}

\author{
Georgios Karamanolis, ${ }^{1,2 *}$ Konstantinos Triantafyllou, ${ }^{1}$ Panagiota Psatha, ${ }_{1}^{1}$ loannis Vlachogiannakos, ${ }^{3}$ Asimina Gaglia, ${ }_{1}^{1}$ \\ Dimitrios Polymeros, ${ }^{1}$ Smaragdi Fessatou, ${ }^{1}$ Maria Triantafyllou, ${ }^{1}$ Ioannis S Papanikolaou ${ }^{1}$ and Spiros D Ladas ${ }^{3}$ \\ ${ }^{1}$ Hepatogastroenterology Unit, 2nd Department of Internal Medicine - Propaedeutic, Attikon University General Hospital, Athens Medical \\ School, Athens, Greece; ' Gastroenterology Unit, 2nd Surgical Department, Aretaieion Hospital, Athens Medical School, Athens, Greece; and \\ ${ }^{3}$ Hepatogastroenterology Unit, 1st Department of Internal Medicine - Propaedeutic, Laikon General Hospital, Athens Medical School, Athens, \\ Greece
}

\section{Background/Aims}

In patients with non-cardiac chest pain (NCCP), gastroesophageal reflux disease (GERD) is the commonest cause and ambulatory $\mathrm{pH}$ is of great value in identifying these patients. However, parameters in the context of predicting therapeutic response are still unknown. By extending the monitoring period, we could better evaluate the best evidence for GERD association. Our aims were (1) to compare the outcomes of 48-hour pH monitoring to 24-hour and (2) to determine whether objective parameters could predict the treatment success in patients with NCCP using Bravo pH system.

\section{Methods}

Pathological esophageal acid reflux (PEAR) and positive symptom index (SI) were calculated after 24-hour and compared to the 48-hour study. Evidence suggestive of GERD diagnosis was considered if PEAR and/or SI (+) were present on each different day. After $\mathrm{pH}$ study, all patients received proton pump inhibitor twice a day for 4 weeks. Treatment success was determined at the end of therapy.

\section{Results}

Thirty-two patients with NCCP participated. GERD was identified in 20 (62.5\%) patients; 17 (53.1\%) had PEAR, 3 (9.4\%) SI $(+)$ and $7(22 \%)$ both. Twelve $(41 \%)$ patients exhibited PEAR values on day 1 , while 17 after 2 days; a $12.1 \%$ gain. SI (+) was found in 6 patients (18.8\%) on day 1 and in 4 more on day 2, a gain of 12.5\%. Significantly higher proportion of patients with GERD indicators showed improvement compared to those without (90\% vs $16.7 \%, P<0.005)$.

\section{Conclusions}

In patients with NCCP, 48-hour pH measurement identified GERD as the cause of NCCP with an increased yield by almost $12 \%$ compared to 12 hours. Objective GERD parameters could predict response to antireflux therapy.

(J Neurogastroenterol Motil 2012;18:169-173)

\section{Key Words}

Bravo; Esophageal pH monitoring; Gastroesophageal reflux

Received: October 29, 2011 Revised: January 4, 2012 Accepted: January 15, 2012

(c) This is an Open Access article distributed under the terms of the Creative Commons Attribution Non-Commercial License (http://creativecommons. org/licenses/by-nc/3.0) which permits unrestricted non-commercial use, distribution, and reproduction in any medium, provided the original work is properly cited.

*Correspondence: Georgios Karamanolis, MD

2nd Department of Internal Medicine - Propaedeutic Attikon University General Hospital, Rimini 1, Haidari, Athens 12462, Greece

Financial support: None.

Tel: +30-210-583-2090, Fax: +30-210-532-6422, E-mail: georgekaramanolis@yahoo.co.uk

Conflicts of interest: None. 


\section{Introduction}

Non-cardiac chest pain (NCCP) is a heterogeneous disorder defined as 'angina-like' chest pain that is not due to ischemic heart disease or other cardiac pathology. ${ }^{1}$ Of those patients who presented to an emergency room with chest pain, a cardiac etiology was ultimately found in only $11 \%$ to $39 \% .^{2,3}$ The esophagus is the most common source of NCCP, with gastroesophageal reflux disease (GERD) constituting up to $60 \%$ of the cases., Typical reflux symptoms were found to be significantly and independently associated with the presence of NCCP. The prevalence of NCCP among patients with frequent and no reflux symptoms was $37.6 \%$ and $12.2 \%$, respectively. ${ }^{5}$

The diagnostic tests available include gastroscopy, esophageal manometry, ambulatory 24-hour esophageal $\mathrm{pH}$ monitoring and an empirical trial with high-dose proton pump inhibitor (PPI). ${ }^{4-7}$ Upper endoscopy has been suggested to have a very limited value in NCCP patients, because of the low prevalence of esophageal mucosal findings. Ambulatory $\mathrm{pH}$ monitoring is particularly helpful in those patients who had normal endoscopy and failed to respond to a therapeutic trial with PPI. ${ }^{1}$ Abnormal $\mathrm{pH}$ testing was documented in $40 \%-50 \%$ of the patients. ${ }^{8,9}$ In patients with $\mathrm{NCCP}$ who had a normal $\mathrm{pH}$ test, calculation of symptom association parameters could increase the sensitivity of the test. $^{10}$

With the evolution of new techniques, such as wireless $\mathrm{pH}$ monitoring, we have gained the advantage of extending the monitoring period upto 48 hours. Thus, the approach appeared to increase the yield for detecting abnormal esophageal acid exposure and the likelihood of establishing a relationship between reflux events and symptoms. ${ }^{11-14}$ However, the value of esophageal parameters in implicating GERD as the etiology of NCCP could be more helpful in predicting the response to antireflux therapy. As far as we know there are no data regarding the evaluation on the effectiveness of NCCP treatment guided by objective parameters determined with wireless ambulatory $\mathrm{pH}$ testing.

The aims of our study were (1) to evaluate the additional yield of 48-hour $\mathrm{pH}$ monitoring compared to 24-hour $\mathrm{pH}$ monitoring using a wireless ambulatory $\mathrm{pH}$ system in patients with NCCP and (2) to determine whether objective esophageal parameters could predict treatment success in these patients.

\section{Materials and Methods}

\section{Study Population}

We included patients who had at least 3 episodes of chest pain per week in the previous 3 months. Patients had undergone a comprehensive diagnostic evaluation by cardiologists in order to exclude a cardiac source for their chest pain. Patients recruited for the study had either normal coronary angiogram or lack of ischemic heart disease on exercise treadmill or stress thallium testing. Informed written consent was obtained from all participated patients. Patients were excluded if they were using aspirin or NSAIDs, had a history of upper gastrointestinal surgery, gastric or duodenal ulcer, connective tissue disease, and severe liver, lung, renal or haematological disease. All patients had a normal esophageal manometry in order to exclude the presence of any esophageal motility disorder.

\section{Ambulatory $\mathrm{pH}$ Monitoring}

Ambulatory esophageal $\mathrm{pH}$ monitoring was performed using the wireless $\mathrm{pH}$ capsule system $\left(\mathrm{BRAVO}^{\mathrm{TM}} \mathrm{pH}\right.$ System; Medtronic Inc., Shoreview, MN, USA). The system allows monitoring for 2 consecutive days. Before each study, the $\mathrm{pH}$ probe was calibrated in buffer solutions of $\mathrm{pH} 7$ and 1 . The probe was deployed at $6 \mathrm{~cm}$ proximal to the squamocolumnar junction, defined by previous upper endoscopy, after transoral introduction using standard placement technique. ${ }^{11,12}$

\section{Study Protocol}

All patients filled out a detailed questionnaire about symptoms and underwent an upper gastrointestinal endoscopy. Ambulatory 24-hour esophageal $\mathrm{pH}$ monitoring was performed on one of the next 7 days. Treatment with PPI was discontinued for at least 15 days; all other drugs potentially affecting gastrointestinal motility and gastrointestinal secretion were discontinued at least 1 week prior to the study.

Data collection device was worn in a belt on the patient's waist. Patients were instructed to resume daily activity and diet. They also recorded the time of meal consumption, indicated the occurrence of symptoms and posture changes on a diary card. Data analysis was performed using standard commercially available computer software program (Polygram ${ }^{\mathrm{TM}}$ NET; Medtronic, Minneapolis, MN, USA). 


\section{Data Analysis}

For the analysis, the periods of meal consumption were marked and excluded. Percentage of time with esophageal $\mathrm{pH}<$ 4 was calculated and pathological esophageal acid reflux (PEAR) was present when esophageal $\mathrm{pH}$ was below 4 for $>5.8 \%$ on day 1 or below $4.5 \%$ on day $2 .^{15}$ The symptom index (SI) was calculated for each patient in relation to acid reflux episodes. Symptom was considered to be associated with reflux if it was preceded within 2 minutes by a reflux episode. ${ }^{16}$ A positive SI was defined if $>50 \%$ of the symptoms were associated with pathological reflux. ${ }^{17}$ If PEAR and/or positive SI were present on either day 1 or day 2, it was considered as an evidence suggestive of GERD diagnosis.

\section{Treatment Schedule}

All patients (regardless of whether having parameters implicating GERD as the etiology of NCCP or otherwise) underwent a therapeutic trial of PPIs twice daily for 4 weeks. The improvement of symptom was assessed by face-to-face interview at the end of antireflux therapy using a 4-point Likert scale $(0=$ no improvement, $1=$ mild improvement, $2=$ moderate improvement and $3=$ marked improvement). Treatment success was defined as the presence of moderate or marked improvement in NCCP symptom.

\section{Statistical Methods}

Values were expressed as mean $\pm \mathrm{SD}$. Statistical analysis was performed by Student's $t$ test or Chi-square testing wherever appropriate. All $P$-values $<0.05$ were considered to be significant.

\section{Results}

\section{Patient Characteristics}

Thirty-two patients (18 men, mean age: $45.3 \pm 12.5$ years) participated in the 2-day ambulatory $\mathrm{pH}$ monitoring. The wireless capsule was successful in all patients who underwent the study. None of the patients required a premature termination of the study or the endoscopic removal of the wireless capsule. Most patients tolerated the wireless $\mathrm{pH}$ capsule well without any discomfort. Four patients (12.5\%) reported symptoms possibly related to capsule placement which included throat pain, ${ }^{3}$ back pain $^{2}$ and globus sensation. ${ }^{1}$

\section{Pathological Esophageal Acid Reflux}

A total of 17 (53.1\%) patients had PEAR. Twelve (41\%) patients exhibited abnormal values on day 1 and 17 (53.1\%) patients on either day of the 2-day study. The overall detection of abnormal esophageal acid exposure was increased by $12.1 \%$ with the addition of day 2 recording.

\section{Symptoms}

Chest pain episodes were reported by 25 (78\%) patients during the study period. Extending the recording time increased the number of patients reporting symptoms from $21(66 \%)$ on day 1 to 25 by the end of the study ( $12 \%$ increase). Moreover, the number of symptoms available for analysis was almost doubled from 24 to 48 hours $(9.2 \pm 2.1$ vs $18.3 \pm 3.9, P<0.001)$.

\section{Symptom-reflux Association}

In $10(31.3 \%)$ patients a positive SI was found; 6 patients (18.8\%) had positive SI on day 1, while 4 more on day 2. A gain of $12.5 \%$ of the patients was observed at the end of the 48 -hour recording. Considering the outcomes of both PEAR and positive SI, a positive SI in conjunction with PEAR was found in 7 (22\%) patients. Addition of SI as an indicator suggesting GERD diagnosis resulted in gaining a total of $3(9.4 \%)$ more patients. Figure showed the prevalence of indicators of GERD on each day of $\mathrm{pH}$ monitoring.

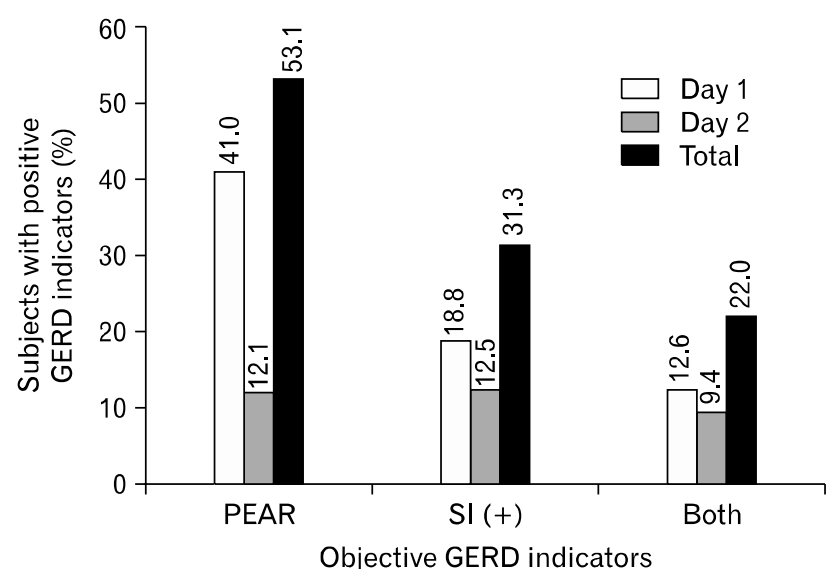

Figure. Prevalence of indicators of GERD on each day of 48-hour wireless ambulatory $\mathrm{pH}$ monitoring. GERD, gastroesophageal reflux disease; PEAR, pathological esophageal acid reflux; SI, symptom index. 


\section{Treatment Response (Objective Pathological Esophageal Parameters Versus Normal Esopha- geal Parameters)}

Of patients with NCCP who had abnormal objective GERD evidence, 18 (90\%) experienced moderate or marked symptom improvement. This contrasts significantly with the group of patients without presence of GERD indicators, where only 2 (16.7\%) patients showed moderate improvement $(P<0.005)$.

Only 2 patients with PEAR as the parameter suggesting GERD did not show improvement at the end of treatment.

\section{Discussion}

Our results showed that over $60 \%$ of study population had evidence of GERD indicators on the ambulatory $\mathrm{pH}$ monitoring. By using a wireless $\mathrm{pH}$ monitoring which extended the recording time to 48 hours we found an increased yield in detection of GERD as the cause of chest pain in these patients. We also improved the detection of abnormal degrees of esophageal acid reflux by extending the $\mathrm{pH}$ recording beyond 24 hours. By the end of the 48 hours study, almost $12 \%$ of patients had evidence of GERD (either PEAR or positive SI) only on day 2. Thus, before the advent of 48 hours wireless $\mathrm{pH}$ testing this proportion of patients would have been identified as negative for GERD and represents the increased diagnostic yield of this technique compared with 24 hours $\mathrm{pH}$ testing. Moreover, we showed that the presence of objective GERD parameters, such as PEAR and/or positive SI could predict a high symptomatic response to therapy with PPIs in patients with NCCP.

Because GERD is by far the most common etiology for $\mathrm{NCCP}$, identification of a GERD-mediated etiology for NCCP is an important process in the evaluation of these patients. Association between chest pain episodes and acid reflux events is considered to reduce repeatitive testing for alternate etiologies, ameliorate patient concerns, and decrease functional disability. ${ }^{2}$ PEAR was found in $53 \%$ of our patients, a figure that was higher than those observed in studies using conventional catheter-based devices. ${ }^{18,19}$ This increased rate represents the additional yield gained by extending the recording period from 1 to 2 days. This increased diagnostic yield was consistent with previously published studies. ${ }^{14,20}$ It showed an almost $10 \%$ gain of pathological acid reflux recording with 48-hour monitoring compared to 24-hour monitoring using a wireless $\mathrm{pH}$ device. The advantages of extending the duration of $\mathrm{pH}$ monitoring were also evident in patients with typical or atypical GERD symptoms. ${ }^{11-13}$

PEAR suggests presence of GERD, but correlation between reflux events and chest pain episodes is required to attribute GERD as the etiology of chest pain. ${ }^{21}$ Symptom index is the most commonly used test for assessing reflux-symptom association. We found that almost $30 \%$ of patients had a positive SI during the study period. This rate was consistent with the previous study using wireless $\mathrm{pH}$ device but was higher compared to the results of a study using catheter-based device. ${ }^{14,22}$ Extending the recording time almost doubled the number of chest pain episodes reported and also increased the number of patients reporting symptoms by $12 \%$. Thus, lengthening $\mathrm{pH}$ monitoring time improved our ability to establish GERD as the cause of chest pain in our study population. A gain of $12.5 \%$ of the patients with a positive SI as GERD indicator was observed at the end of the 48-hour recording. Although the majority of patients with a positive SI had also pathological acid reflux, addition of SI resulted in an increase of detection of patients with evidence of GERD by $10 \%$. The combination of a positive SI and pathological acid reflux on wireless $\mathrm{pH}$ monitoring identified $62.5 \%$ of patients with evidence linking GERD to NCCP.

According to most recently published review regarding NCCP, after exclusion of cardiac origin pain, twice-daily PPI should be tried before other invasive tests including ambulatory $\mathrm{pH}$ testing. ${ }^{23}$ However, in patients with unselected GERD symptoms, the specificity of a PPI trial for a GER diagnosis has been reported as low as $54 \% .{ }^{24} \mathrm{It}$ is also known that some patients with NCCP failed to response to short term treatments with PPIs. ${ }^{25}$ For these reasons, ambulatory $\mathrm{pH}$ testing is of great value in determining GERD as the cause of NCCP. Identification of abnormal objective $\mathrm{pH}$ parameters, including PEAR and positive SI emphasize also the value of $\mathrm{pH}$ monitoring in predicting therapeutic success in patients with NCCP. Indeed, our study showed that marked improvement of symptoms was observed in $90 \%$ of patients with abnormal objective GERD indicators. In contrast, only $17 \%$ of patients without relevant GERD parameters showed improvement after PPI treatment. Our result is in agreement with a recent study showing that response to antireflux therapy was best predicted when GERD parameters were abnormal and poorest when parameters were normal. ${ }^{10}$

In conclusion, our study showed that combination of a positive SI and pathological acid reflux could identify GERD as the etiology of chest pain in $62.5 \%$ of patients with NCCP. By extending the period of $\mathrm{pH}$ monitoring to 2 days using a wireless $\mathrm{pH}$ device we observed an increased yield in detecting evidence 
of GERD in patients with NCCP by almost $12 \%$. Presence of objective abnormal GERD parameters could better predict response to PPI therapy compared to presence of normal GERD indicators.

\section{References}

1. Faybush EM, Fass R. Gastroesophageal reflux disease in noncardiac chest pain. Gastroenterol Clin North Am 2004;33:41-54.

2. Eslick GD, Jones MP, Talley NJ. Non-cardiac chest pain: prevalence, risk factors, impact and consulting: a population-based study. Aliment Pharmacol Ther 2003;17:1115-1124.

3. Pope JH, Aufderheide TP, Ruthazer R, et al. Missed diagnoses of acute cardiac ischemia in the emergency department. N Engl J Med 2000;342:1163-1170.

4. Richter JE. Chest pain and gastroesophageal reflux disease. J Clin Gastroenterol 2000;30:S39-S41.

5. Chiocca JC, Olmos JA, Salis GB, et al. Prevalence, clinical spectrum and atypical symptoms of gastro-oesophageal reflux in Argentina: a nationwide population-based study. Aliment Pharmacol Ther 2005; 22:331-342.

6. Fass R, Fennerty MB, Ofman JJ, et al. The clinical and economic value of a short course of omeprazole in patients with noncardiac chest pain. Gastroenterology 1998;115:42-49.

7. Hsia PC, Maher KA, Lewis JH, Cattau EL Jr, Fleischer DE, Benjamin SB. Utility of upper endoscopy in the evaluation of noncardiac chest pain. Gastrointest Endosc 1991;37:22-26.

8. Fass R, Naliboff B, Higa L, et al. Differential effect of long-term esophageal acid exposure on mechanosensitivity and chemosensitivity in humans. Gastroenterology 1998;115:1363-1373.

9. Beedassy A, Katz PO, Gruber A, Peghini PL, Castell DO. Prior sensitization of esophageal mucosa by acid reflux predisposes to reflux-induced chest pain. J Clin Gastroenterol 2000;31:121-124.

10. Kushnir KW, Sayuk GS, Gyawali PC. Abnormal GERD parameters on ambulatory $\mathrm{pH}$ monitoring predict therapeutic success in noncardiac chest pain. Am J Gastroenetrol 2010;105:1032-1038.

11. Pandolfino JE, Richter JE, Ours T, Guardino JM, Chapman J, Kahrilas PJ. Ambulatory esophageal $\mathrm{pH}$ monitoring using a wireless system. Am J Gastroenterol 2003;98:740-749.

12. Ward EM, Devault KR, Bouras EP, et al. Successful esophageal pH monitoring with a catheter-free system. Aliment Pharmacol Ther
2004;19:449-454

13. Prakash C, Clouse RE. Value of extended recording time with wireless $\mathrm{pH}$ monitoring in evaluating gastroesophageal reflux disease. Clin Gastroenterol Hepatol 2005;3:329-334.

14. Prakash $\mathrm{C}$, Clouse $\mathrm{RE}$. Wireless $\mathrm{pH}$ monitoring in patients with non-cardiac chest pain. Am J Gastroenterol 2006;101:446-452.

15. Ayazi S, Lipham J, Portale G, et al. Bravo catheter free $\mathrm{pH}$ monitoring: normal values, concordance, optimal diagnostic thresholds and accuracy. Clin Gastroenterol Hepatol 2009;7:60-67.

16. Lam HG, Breumelhof R, Roelofs JM, Van Berge Henegouwen GP Smout AJ. What is the optimal time window in symptom analysis of 24-hour esophageal pressure and pH data? Dig Dis Sci 1994;39:402409.

17. Bredenoord AJ, Weusten BL, Smout AJ. Symptom association analysis in ambulatory gastro-oesophageal reflux monitoring. Gut 2005; 54:1810-1817.

18. Hewson EG, Sinclair JW, Dalton CB, Richter JE. Twenty-fourhour esophageal $\mathrm{pH}$ monitoring: the most useful test for evaluating noncardiac chest pain. Am J Med 1991;90:576-583.

19. Ho KY, Ng WL, Kang JY, Yeoh KG. Gastroesophageal reflux disease is a common cause of noncardiac chest pain in a country with a low prevalence of reflux esophagitis. Dig Dis Sci 1998;43:19911997.

20. Garrean CP, Zhang Q, Gonsalves N, Hirano I. Acid reflux detection and symptom-reflux association using 4-day wireless $\mathrm{pH}$ recording combining 48-hour periods off and on PPI therapy. Am J Gastroenterol 2008;103:1631-1637.

21. Pandolfino JE, Vela MF. Esophageal-reflux monitoring. Gastrointest Endosc 2009;69:917-930.

22. Dekel R, Martinez-Hawthorne SD, Guillen RJ, Fass R. Evaluation of symptom index in identifying gastroesophageal reflux disease-related noncardiac chest pain. J Clin Gastroentrol 2004;38:24-29.

23. Kahrilas PJ, Smout AJ. Esophagel disorders. Am J Gastroenterol 2010;105:747-756.

24. Numans ME, Lau J, de Wit NJ, Bonis PA. Short-term treatment with proton-pump inhibitors as a test for gastroesophageal reflux disease: a meta-analysis of diagnostic test characteristics. Ann Intern Med 2004;140:5 18-527.

25. Gasiorowska A, Fass R. The proton pump inhibitor (PPI) test in GERD: does it still have a role? J Clin Gastroenterol 2008;42:867874. 